\title{
Gravitational waves in the Hyperspace?
}

\author{
Christian Corda*, Giorgio Fontana ${ }^{+}$and Gloria Garcia Cuadrado\&
}

June 12, 2021

\begin{abstract}
*Associazione Scientifica Galileo Galilei, Via Pier Cironi 16 - 59100 Prato, Italy and 0574news.it - Sezione Scientifico-Tecnologica, via Sante Pisani 46 - 59100 Prato, Italy; ${ }^{+}$Università di Trento, 38050 Povo (Trento), Italy; ${ }^{\&}$ Aerospace Tecnology Center, Av. Segle XXI - E-08840 Viladecans (Barcelona), Spain

\author{
E-mail addresses: * christian.corda@ego-gw.it; ${ }^{+}$giorgio.fontana@unitn.it; \\ gloria.garcia@ctae.org
}

\begin{abstract}
In the framework of the debate on high-frequency gravitational waves (GWs), after a review of GWs in standard General Relativity, which is due for completness, the possibility of merging such a traditional analysis with the Hyperspace formalism that has been recently introduced in some papers in the literature, with the goal of a better understanding of manifolds dimensionality also in a cosmological framework, is discussed. Using the concept of refractive index in the Hyperspace, spherical solutions are given and the propagation of GWs in a region of the Hyperspace with an unitary refractive index is also discussed. Propagation phenomena associated to the higher dimensionality are proposed, possibly including non-linear effects. Further and accurate studies in this direction are needed.
\end{abstract}

- PACS numbers: 04.80.Nn, 04.30.Nk, 04.30.-w

- Keywords: gravitational waves; General Relativity; Hyperspace; Cosmology.

The data analysis of interferometric GWs detectors has recently been started (for the current status of GWs interferometers see [1, 2, 3, 4, 5, 6, 7, 8, ) and the scientific community aims at a first direct detection of GWs in next years.

Detectors for GWs will be important for a better knowledge of the Universe and either to confirm or ruling out the physical consistency of General Relativity or any other theory of gravitation [9, 10, 11, 12, 13, 14. In fact, in the context of Extended Theories of Gravity, some differences between General Relativity 
and the other theories can be pointed out starting from the linearized theory of gravity [9, 10, 12, 14].

Recently, some papers in the literature have shown the importance of highfrequency GWs 15, 16, 17. In this context, a difference approach to gravity has also been proposed with the Hyperspace formalism, performing a coordinatetransformation from spacetime to Euclidean coordinates [18, 19.

After a review of GWs in standard General Relativity, which is due for completness, in this letter we discuss the possibility of merging such a traditional analysis with the Hyperspace formalism that has been recently introduced in some papers in the literature with the goal of a better understanding of manifolds dimensionality also in a cosmological framework [18, 19, 20]. Using the concept of refractive index in the Hyperspace, we find spherical solutions and we also discuss the propagation of GWs in a region of the Hyperspace with an unitary refractive index.

After this, propagation phenomena associated to the higher dimensionality are proposed, possibly including non-linear effects. Further and accurate studies in this direction are needed.

Gravitational waves in General Relativity have been analyzed in lots of works in literature, starting by the work of the Bondi's research group [20] expecially in the Transverse-Traceless (TT) gauge [19, 20, 21. In the first part of this letter, we reanalyze the TT gauge for GWs following [28].

In the context of General Relativity, working with $c=1$ and $\hbar=1$, calling $\widetilde{R}_{\mu \nu \rho \sigma}, \widetilde{R}_{\mu \nu}$ and $\widetilde{R}$ the linearized quantity which correspond to $R_{\mu \nu \rho \sigma}, R_{\mu \nu}$ and $R$, and putting

$$
g_{\mu \nu}=\eta_{\mu \nu}+h_{\mu \nu} \text { with }\left|h_{\mu \nu}\right| \ll 1
$$

the linearized Einstein field equations can be written like [20, 24]

$$
\widetilde{R}_{\mu \nu}-\frac{\widetilde{R}}{2} \eta_{\mu \nu}=0
$$

Defining

$$
\bar{h}_{\mu \nu} \equiv h_{\mu \nu}-\frac{h}{2} \eta_{\mu \nu}
$$

and putting eq. (3) in eqs. (2) it is

$$
\square h_{\mu \nu}-\partial_{\mu}\left(\partial^{\alpha} \bar{h}_{\alpha \nu}\right)-\partial_{\nu}\left(\partial^{\alpha} \bar{h}_{\alpha \mu}\right)+\eta_{\mu \nu} \partial^{\beta}\left(\partial^{\alpha} \bar{h}_{\alpha \beta}\right),
$$

where $\square$ is the D'Alembertian operator.

Now, let us consider the gauge transform (Lorenz condition)

$$
\bar{h}_{\mu \nu} \rightarrow \bar{h}_{\mu \nu}^{\prime}=\bar{h}_{\mu \nu}-\partial_{(\mu} \epsilon_{\nu)}+\eta_{\mu \nu} \partial^{\alpha} \epsilon_{\alpha},
$$

with the condition $\square \epsilon_{\nu}=\partial^{\mu} \bar{h}_{\mu \nu}$ for the parameter $\epsilon^{\mu}$. It is

$$
\partial^{\mu} \bar{h}_{\mu \nu}^{\prime}=0
$$


and, omitting the ', the field equations can be rewritten like

$$
\square \bar{h}_{\mu \nu}=0 .
$$

Solutions of eqs. (77) are plane waves, i.e.

$$
\bar{h}_{\mu \nu}=A_{\mu \nu}(\vec{k}) \exp \left(i k^{\alpha} x_{\alpha}\right)+c . c .
$$

which take the conditions

$$
\begin{aligned}
k^{\alpha} k_{\alpha} & =0 \\
k^{\mu} A_{\mu \nu} & =0,
\end{aligned}
$$

which arises respectively from the linearized field equations and from eq. (6). The first of eqs. (9) shows that perturbations have the speed of the light, the second represents the transverse property of the waves.

Fixed the Lorenz gauge, another transformation with $\square \epsilon^{\mu}=0$ can be performed; one takes

$$
\begin{gathered}
\square \epsilon^{\mu}=0 \\
\partial_{\mu} \epsilon^{\mu}=0,
\end{gathered}
$$

which works because $\square \bar{h}=0$. Then

$$
\bar{h}=0 \Rightarrow \bar{h}_{\mu \nu}=h_{\mu \nu},
$$

(traceless property) i.e. $h_{\mu \nu}$ is a transverse plane wave too. The gauge transformation (10) also saves the conditions

$$
\begin{gathered}
\partial^{\mu} \bar{h}_{\mu \nu}=0 \\
\bar{h}=0 .
\end{gathered}
$$

Considering a wave incoming in the positive $z$ direction it is

$$
k^{\mu}=(k, 0,0 k)
$$

and the second of eqs. (9) implies

$$
\begin{gathered}
A_{0 \nu}=-A_{3 \nu} \\
A_{\nu 0}=-A_{\nu 3} \\
A_{00}=-A_{30}+A_{33} .
\end{gathered}
$$

Now, one has to compute the freedom degrees of $A_{\mu \nu}$. As $A_{\mu \nu}$ is a symmetric tensor, 10 components were present at the begining of the analysis. 3 components have been lost for the transverse property, more, the condition (11) 
reduces the components to 6 . One can take $A_{00}, A_{11}, A_{22}, A_{21}, A_{31}, A_{32}$ like independent components; another gauge freedom can be used to put to zero three more components (i.e. one can only chose three of $\epsilon^{\mu}$, the fourth component depends from the others by $\left.\partial_{\mu} \epsilon^{\mu}=0\right)$.

Then, taking

$$
\begin{gathered}
\epsilon_{\mu}=\tilde{\epsilon}_{\mu}(\vec{k}) \exp \left(i k^{\alpha} x_{\alpha}\right)+\text { c.c. } \\
k^{\mu} \tilde{\epsilon}_{\mu}=0,
\end{gathered}
$$

the transform law for $A_{\mu \nu}$ is (see eqs. (5) and (8) )

$$
A_{\mu \nu} \rightarrow A_{\mu \nu}^{\prime}=A_{\mu \nu}-2 i k\left({ }_{\mu} \tilde{\epsilon}_{\nu}\right) .
$$

As for the six components of interest only three are gauge-invariants, $\tilde{\epsilon}_{\nu}$ can be chosen to put equal to zero the others (see 28] for details). From the traceless property two components are also equal 28. In this way, only two physical components are present and the total perturbation of a gravitational wave propagating in the $z+$ direction in this gauge is [28]

$$
h_{\mu \nu}(t-z)=A^{+}(t-z) e_{\mu \nu}^{(+)}+A^{\times}(t-z) e_{\mu \nu}^{(\times)},
$$

that describes the two polarizations of gravitational waves which arises from General Relativity in the transverse-traceless (TT) gauge (ref. [20, 19, 20]). This gauge is historically called TT, because in these particular coordinates the gravitational waves have a transverse effect and are traceless.

A different approach on gravity has been recently proposed considering the Hyperspace formalism, performing a coordinate-transformation from spacetime to Euclidean coordinates [18, 19. In this framework, a different space-time structure is introduced [16, 18, 19, 25, 22,. One starts by the flat space-time line element

$$
d \tau^{2}=-d t^{2}+d z^{2}+d x^{2}+d y^{2},
$$

and re-arranges like [16, 18, 19, 25, 22,

$$
d t^{2}=d \tau^{2}+d z^{2}+d x^{2}+d y^{2} .
$$

Gravity is considered by resorting the analogy to optical propagation in the 3 -space. In this way, a gravitational refractive index of the Hyperspace named $n$ is introduced [25, 27]

$$
d t^{2}=n^{2}\left(d \tau^{2}+d z^{2}+d x^{2}+d y^{2}\right) .
$$

The case $n=1$ gives the Lorentzian flat space-time (18), while, switching to spherical coordinates and putting $d \theta=d \varphi$, the condition [25, 27]

$$
n=\left(1-\frac{2 \tilde{G} m}{r}\right)^{-1}
$$


generates the well known Schwarzschild space-time [20].

One can also introduce directional indexes of the Hyperspace

$$
\left.d t^{2}=n_{\tau}^{2} d \tau^{2}+n_{z}^{2} d z^{2}+n_{x}^{2} d x^{2}+n_{y}^{2} d y^{2}\right),
$$

and, recalling that the GWs line element which arises by eq. (17), considering only the "+" polarization, is [20, 28]

$$
d \tau^{2}=-d t^{2}+d z^{2}+\left[1+A^{+}(t-z)\right] d x^{2}+\left[1-A^{+}(t-z)\right] d y^{2},
$$

one obtains

$$
\begin{gathered}
n_{\tau}^{2}=1 \\
n_{z}^{2}=1 \\
n_{x}^{2}=1+A^{+}(t-z) \\
n_{y}^{2}=1-A^{+}(t-z) .
\end{gathered}
$$

More in general, assuming an isotropic propagation, one consider a generic wave equation for weak waves travelling at the speed of light 25]

$$
\square n=0 .
$$

In Hyperspace coordinates, by separing variables, one looks for solutions of the type $n(r, t)=R(r) T(T)$, obtaining

$$
\begin{gathered}
r \frac{d^{2} R}{d r^{2}}+3 \frac{d R}{d r}+k^{2} r R=0 \\
\frac{d^{2} T}{d t^{2}}+\omega^{2} T=0 .
\end{gathered}
$$

In the flat background the refractive index is unitary. To study non linear effects one has to include such a value in the expression of the speed. In this way eq. (25) changes into

$$
(n+1)^{2} \frac{\partial^{2} n}{\partial t^{2}}=\nabla^{2} n=0,
$$

which can be re-written like

$$
\square n=-\left(n^{2}+2 n\right) \frac{\partial^{2} n}{\partial t^{2}} .
$$

Eq. (25) is re-obtained in the limit $n \ll 1$.

These equations show that if a beam of GWs is more intense at center than at wings, the central part travels at lower four-speed (self-focusing, see also the classical tratment in [29]).

The unitary background refractive index is attributed to a cosmological GWs background which supports the spacetime 25 . The existence of such a cosmological GWs background has been postulated by lots works in the literature, see 
for example [30, 23, 24, 25] and it has been recently analysed in the framework of Extended Theories of Gravity [34, 35, 36.

Removing the background, the non-linear eq. (27) becomes

$$
n^{2} \frac{\partial^{2} n}{\partial t^{2}}=\nabla^{2} n=0 .
$$

In this case, solutions of the type $n(r, t)=R(r) T(T)$ give 25]

$$
\begin{gathered}
r \frac{d^{2} R}{d r^{2}}+3 \frac{d R}{d r}-\varkappa r R^{2}=0 \\
\frac{d^{2} T}{d t^{2}}+\frac{-\varkappa}{T}=0 .
\end{gathered}
$$

The second of eqs. (30) is a non linear differential equation of the EmdenFowler type 37, which can be integrated only numerically in terms of $T(t)$ if $\varkappa \neq 0$. It is possible to write

$$
t=C_{1}+\int d t(2 \varkappa \ln T)^{-\frac{1}{2}}
$$

where $C_{1}$ and $C_{2}$ are two real integration constants which work in the numerical integration

$$
T(t) \approx C_{1}+C_{2} t^{\alpha}
$$

and $\alpha \approx 1.03$. Note: solution with both of $C_{1} \neq 0$ and $C_{2}=0$ are not acceptable as $T(t)=$ constant is not solution of the second of eqs. (30). In such a numerical integration the slope and the sign are given by initial conditions and the equation is singular in the case $C_{1}=0$.

With $\varkappa=0$ one gets $T(t)=C_{1}+C_{2} t$, while with $\varkappa<0$ the solution of the first of eqs. (30) is a damped oscillator.

A possible interpretation of the results [25] is that the large scale sweeping of spacetimes through the Hyperspace becomes slower and slower with time, while, because of the increasimg temporal component of $n$, the oscillating and decreasing spatial component of $n$, combined with a specific spacetime motion results in a nearly constant $n$ for the local background, during the spacetime evolution [25]. The $r$-dependence in the first of eqs. (30) implies that spacetime regions in which the particles propagation speed can be different from the speed of the light, and the presence of Hyperspace "highways" is not totally excluded. Further and accurate studies are needed in this direction.

In the Hyperspace $n$ can also oscillate from positive to negative values, and further studies will be needed to understand the effect of the sign change too.

$\varkappa=0$ gives also $R(r)=-3 \ln r+C_{3}$ [25, thus, in this particular case the solution of eqs. (30) is

$$
n(r, t)=\left(C_{1}+C_{2} t\right)\left(-3 \ln r+C_{3}\right) .
$$

Again, this particular solution shows that the speed of spacetimes changes in the Hyperspace because both of evolution in time and expansion in space. 


\section{Conclusion remarks}

In the framework of the debait on high-frequency GWs, after a review of GWs in standard General Relativity, which is due for completness, the possibility of merging such a traditional analysis with the Hyperspace formalism which has been recently introduced in some papers in the literature, with the goal of a better understanding of manifolds dimensionality also in a cosmological framework, has been discussed. Using the concept of refractive index in the Hyperspace, spherical solutions have been given and the propagation of GWs in a region of the Hyperspace with an unitary refractive index has been also discussed. Propagation phenomena associated to the higher dimensionality have been proposed, possibly including non-linear effects. Further and accurate studies in this direction are needed.

\section{Aknlowdgements}

This letter has been partially supported by the Sezione Scientifico-Tecnologica of 0574news.it, via Sante Pisani 46, 59100 Prato, Italy

\section{References}

[1] F. Acernese et cal. (the Virgo Collaboration) - Class. Quant. Grav. 24, 19, S381-S388 (2007)

[2] C. Corda - Astropart. Phys. 27, No 6, 539-549 (2007)

[3] C. Corda - Int. J. Mod. Phys. D 16, 9, 1497-1517 (2007)

[4] B. Willke et al. - Class. Quant. Grav. 23 8S207-S214 (2006)

[5] D. Sigg (for the LIGO Scientific Collaboration) www.ligo.org/pdf_public/P050036.pdf

[6] B. Abbott et al. (the LIGO Scientific Collaboration) - Phys. Rev. D 72, $042002(2005)$

[7] M. Ando and the TAMA Collaboration - Class. Quant. Grav. 197 1615$1621(2002)$

[8] D. Tatsumi, Y. Tsunesada and the TAMA Collaboration - Class. Quant. Grav. 215 S451-S456 (2004)

[9] S. Capozziello - Newtonian Limit of Extended Theories of Gravity in Quantum Gravity Research Trends Ed. A. Reimer, pp. 227-276 Nova Science Publishers Inc., NY (2005) - also in arXiv:gr-qc/0412088 (2004);E. Elizalde, S. Nojiri, and S.D. Odintsov - Phys. Rev. D 70, 043539 (2004)

[10] S. Capozziello and A. Troisi - Phys. Rev. D 72044022 (2005);G. Cognola, E. Elizalde, S. Nojiri, S.D. Odintsov and S. Zerbini - J. Cosmol. Astropart. Phys. JCAP0502(2005)010 
[11] G. Allemandi, M. Capone, S. Capozziello and M. Francaviglia - Gen. Rev. Grav. 381 (2006); G. Allemandi, M. Francaviglia, M. L. Ruggiero and A . Tartaglia - Gen. Rel. Grav. 3711 (2005)

[12] S. Capozziello and C. Corda - Int. J. Mod. Phys. D 151119 -1150 (2006);S. Nojiri and S.D. Odintsov - hep-th 0601213 (2006)

[13] G. Cognola, E. Elizalde, S. Nojiri, S.D. Odintsov and S. Zerbini - Phys. Rev. D 73, 084007 (2006)

[14] C. Corda- Astropart. Phys. 28, 247-250 (2007);G. Cognola, E. Elizalde, S. Nojiri, S.D. Odintsov, L. Sebastiani, S. Zerbini - Phys. Rev. D 77, 046009 (2008)

[15] F. Li, R.M.L. Baker Jr., Z. Fang, G. V. Stephenson and Z. Chen arXiv:0806.1989, accepted for publication by Eur. Phys. Journ. C

[16] G. Fontana, P. Murad and R.M.L. Baker Jr. - AIP Conference Proceedings 880, 1117-1124, Melville, New York (2007)

[17] P. Murad and R.M.L. Baker Jr. - $39^{h}$ AIAA/ASME/SAE/ASEE Propulsion Conference Proceedings N. 2003-4882, Hunsville, Alabama, USA (2003)

[18] J.M.C. Mountanus - Found. of Phys. 31, 1357-1359 (2001)

[19] A. Gersten - Found. of Phys. 33, 1237-1251 (2003)

[20] Bondi H, Pirani FAE and Robinson I - Proc. Roy. Soc. Lond. A251 519-533 (1959)

[19] Rakhmanov M - Phys. Rev. D 71084003 (2005)

[20] Misner CW, Thorne KS and Wheeler JA - "Gravitation" - W.H.Feeman and Company - 1973

[21] Maggiore M- Physics Reports 331 283-367 (2000)

[24] Landau L and Lifsits E - "Teoria dei campi" - Editori riuniti edition III (1999)

[25] G. Fontana - AIP Conference Proceedings 978-0-7354-0486, Melville, New York (2008)

[22] G. Fontana - AIP Conference Proceedings 746, 1403 - 1410, Melville, New York (2005)

[27] J. B. Almeida - gr-qc/040722

[28] C. Corda - Int. Journ. Mod. Phys. A 22, 26, 4859-4881 (2007)

[29] V. Ferrari - Phys. Rev. D 37, 10, 3061-3064 (1988) 
[30] B. Allen - Proceedings of the Les Houches School on Astrophysical Sources of Gravitational Waves, eds. Jean-Alain Marck and Jean-Pierre Lasota (Cambridge University Press, Cambridge, England 1998).

[23] B. Allen - Phys. Rev. D 37, 2078 (1988)

[24] L. P. Grishchuk and others - Phys. Usp. 44 1-51 (2001)

[25] L. P. Grishchuk and others - Usp. Fiz. Nauk 1713 (2001)

[34] C. Corda, S. Capozziello and M. F. De Laurentis - AIP Conference Proceedings, Volume 966, pp 257-263 (2007) - Proceedings of the Fourth ItalianSino Workshop on Relativistic Astrophysics July 20-30 2007, Pescara, Italy

[35] S. Capozziello, C. Corda and M. F. De Laurentis - Mod. Phys. Lett. A 22, $35,2647-2655(2007)$

[36] C. Corda - Astropart. Phys., 30, 209-215 (2008)

[37] P. L. Sachdev - Nonlinear ordinary differential equations and their applications, Marcel Dekker, Inc., New York, 1991 